data clearly confirm the identification of RF as a potent amplifier of the FcR-dependent inflammatory reaction induced in macrophages by the tightly RA-associated ACPA-IC. They clarify the link between disease aggressiveness and both RF and ACPA.

\section{A88 CYTOKINE PROFILE OF MACROPHAGES IN VITRO STIMULATED BY ACPA IMMUNE COMPLEXES IN THE PRESENCE OR ABSENCE OF IGM RHEUMATOID FACTOR}

Lætitia Laurent, ${ }^{1}$ Cyril Clavel, ${ }^{1,2}$ Florence Anquetil, ${ }^{1}$ Jean-Louis Pasquali, ${ }^{3}$ Mireille Sebbag, ${ }^{1}$ Guy Serre', ${ }^{1}$ Laboratory of Epidermis Differentiation and Rheumatoid Autoimmunity, CNRS-Université de Toulouse, France; 'Laboratory of Cell Biology and Cytology, Institut Fédératif de Biologie, CHU de Toulouse, Toulouse, France; ${ }^{3}$ Laboratory of Immunology and Therapeutical Chemistry, CNRS, Strasbourg, France

10.1136/ard.2010.148973.6

Background Autoantibodies to citrullinated proteins (ACPA) are suspected to play a central role in the pathophysiology of rheumatoid arthritis (RA). By stimulating monocyte-derived macrophages from healthy donors with ACPA-containing immune complexes (ACPA-IC), the authors previously established the inflammatory potential of ACPA through FcrRIIa engagement. Furthermore, the authors evidenced a major enhancement of tumour necrosis factor $\alpha$ (TNF $\alpha)$ secretion when these IC also included monoclonal IgM rheumatoid factors (RFs) derived from patients with cryoglobulinemia (ACPA/RF-IC).

Objectives To further investigate the inflammatory effect of ACPA-IC including or not RF, the authors evaluated the response of blood monocytes to ACPA/RF-IC and specified the cytokine profile induced in macrophages by ACPA-IC and ACPA/RF-IC.

Materials and methods Three monoclonal IgM RF were purified from the serum of patients with mixed cryoglobulinemia. Monocyte purification, macrophage differentiation and macrophage stimulation by IC in the absence of complement were performed as previously described (Clavel et al, Arthritis Rheum, 2008). In culture supernatants, TNF $\alpha$ was assayed by ELISA, and interleukin $1 \beta$ (IL-1 $\beta$ ), IL-6, IL-8, IL-10 and IL-1Ra were measured in a multiplexed flow cytometric assay.

Results Stimulation by ACPA-IC and ACPA/RF-IC was compared in monocyte and macrophage pairs derived from three donors. While, as expected, in macrophages, TNF $\alpha$ secretions were higher after stimulation by ACPA/RF-IC, in monocytes only very low TNF $\alpha$ secretions were detected in both conditions. Stimulations with immobilised and IgG-conjugated RF in four monocyte and macrophage pairs confirmed these results. In macrophages from eight blood donors, in addition to inducing TNF $\alpha$, ACPA-IC also importantly raised IL-8 secretion while no significant secretions of IL-1 $\beta$, IL- 6 or IL-10 were detected. Secretion of IL-1Ra was found to be enhanced in two out of two donors tested. For three of the eight donors the macrophages were additionally stimulated with ACPA/RF-IC. In all these donors, compared to stimulation with ACPA-IC, IL-10 secretion was enhanced. However, this was compensated by an important upregulation of TNF $\alpha$, by the induction of IL-1 $\beta$ and IL- 6 secretion, and persistence of high IL-8 levels. Moreover, IL-1Ra was down modulated in two out of two tested donors.

Conclusions Monocytes are refractory to stimulation by IgM RF-containing IC, suggesting that circulating $\operatorname{Ig} M$ RF-containing IC remain fairly innocuous as far as FcR engagement of circulating monocytes is concerned. In addition, this 